\title{
Deep venous thrombosis of the arm: a study of coagulation and fibrinolysis
}

\author{
S-B SUNDQVIST, U HEDNER, H K E KULLENBERG， S-E BERGENTZ
}

\begin{abstract}
Sixty consecutive patients with phlebographically verified deep venous thrombosis of the upper arm were studied for disorders of coagulation and fibrinolysis. No appreciable increase in abnormalities of the factor VIII complex, antithrombin III, or inhibitors of activators of fibrinolysis were found. A decreased fibrinolytic defence mechanism, evident either as a deficient release capacity of fibrinolytic activators from the vein during stasis or as decreased fibrinolytic activity in the vein wall as determined histochemically, was found in 26 out of 53 patients studied (49\%).

It is concluded that deep venous thrombosis of the upper arm is a multifactorial disease. An impaired fibrinolytic defence mechanism is one of the factors that may be of pathogenetic importance.
\end{abstract}

\section{Introduction}

Deep venous thrombosis of the upper arm may affect the axillary vein or subclavian vein or both. It is a rare event, with an incidence of about $1-2 \%$ of that of deep venous thrombosis of the leg. ${ }^{12}$ The peak incidence occurs at 30-40 years of age, the male to female ratio is about $3: 2$, the right arm seems to be affected more often than the left, and the condition is rarely seen as a postoperative complication. ${ }^{3}$

The condition was first described by Sir James Paget ${ }^{4}$ and von Schroetter ${ }^{5}$ and is sometimes referred to as Paget-von Schroetter's syndrome. It has been given several other names, including

\footnotetext{
Department for Coagulation Disorders, University of Lund, Malmö General Hospital, S-214 01 Malmö, Sweden

S-B SUNDQVIST, MD, registrar

U HEDNER, MD, associate professor, consultant

Department of Surgery, University of Lund

H K E KULLENBERG, MD, registrar

S-E BERGENTZ, $M D$, professor of surgery
}

effort thrombosis and primary thrombosis of the axillary veins, ${ }^{16-8}$ indicating that the aetiology is probably multifactorial and not well known. Trauma to the axillary vein has been claimed to be the most important aetiological factor, but mild but repetitive trauma to the vessels, suppposed to occur in various unaccustomed, heavy activities, has also been claimed to be aetiologically important. ${ }^{3}>9$ Other factors, such as stasis of the arm vessels, may also be important, as they are for development of deep venous thrombosis elsewhere in the body. The condition is sometimes associated with congestive heart failure, particularly when it occurs on the left side. ${ }^{13}$

In patients with so-called spontaneous deep venous thrombosis in the lower legs defective fibrinolytic capacity in the vessel wall has been reported as a common finding. ${ }^{10}$ No studies of coagulation and fibrinolysis in patients with thrombosis in the upper arm have been reported. We report on a series of 60 patients with axillary vein thrombosis in whom detailed studies of coagulation and the fibrinolytic system were carried out.

\section{Patients and methods}

We studied 60 consecutive patients ( 36 women and 24 men) referred to this hospital because of thrombosis of a subclavian or axillary vein. In each case the diagnosis was verified by phlebography. Fifty-three of the patients had been referred from other hospitals to the Coagulation Laboratory in Malmö, which is the only specialised laboratory for coagulation analysis in southern Sweden. A few patients had been referred to the department of surgery for evaluation as the thoracic outlet syndrome was suspected.

The right arm was affected in 37 cases, the left in 21 , and both arms in two. The mean age of the men was 32.4111 .7 years (distribution 16-75 years) and of the women 33.9 9.1 years (distribution 17-59 years).

Factors that may be important for the development of thrombosis were found on 44 occasions ( 34 patients). A history of thrombotic disease in the lower leg was found in six patients, including one with bilateral subclavian vein thrombosis. A family history of thrombosis was found in eight patients. In two patients the thrombosis had developed after trauma to the shoulder, one of the patients having sustained a fractured clavicle one week before the symptoms started. Five patients had been doing extensive and heavy work just before the onset of symptoms. This included long-distance swimming and work in a forest and a factory. All five patients, however, were 
accustomed to such work. Two patients had a cervical rib with neurological symptoms and later underwent transaxillary rib resection. One additional patient, who did not have a cervical rib, underwent operation for the thoracic outlet syndrome. Ten patients were taking contraceptive pills: of the nine women aged between 15 and 24 years, four were taking the pill; of the nine between 25 and 34 years, three were taking the pill; and of the seven between 35 and 44 years, two were taking the pill. Two patients were pregnant when the thrombosis occurred. One patient had cancer of the breast with a metastasis in the axilla on the affected side. One patient had cancer of the prostatic gland. Two patients developed the thrombosis during intensive care, one after operation for pancreatitis and the other during treatment for hepatic coma. Two patients had severe myocardial insufficiency, one with pneumonia in addition. Two patients were alcoholics.

Control subjects comprised 108 adult healthy volunteers (29 women and 79 men) with an average age of 34.2 years (range 20-75 years; range among the women 25-71 years and among the men $20-75$ years).

\section{LABORATORY METHODS}

The coagulation and fibrinolytic studies were performed on average two to three months after the thrombotic episode to avoid any influence of the acute reactive process.

The following variables were determined: factor VIII clotting activity, factor VIII-related antigen, antithrombin III (immunochemical method), and inhibitors of activation of plasminogen. The procedures used have been described elsewhere. ${ }^{11-15}$

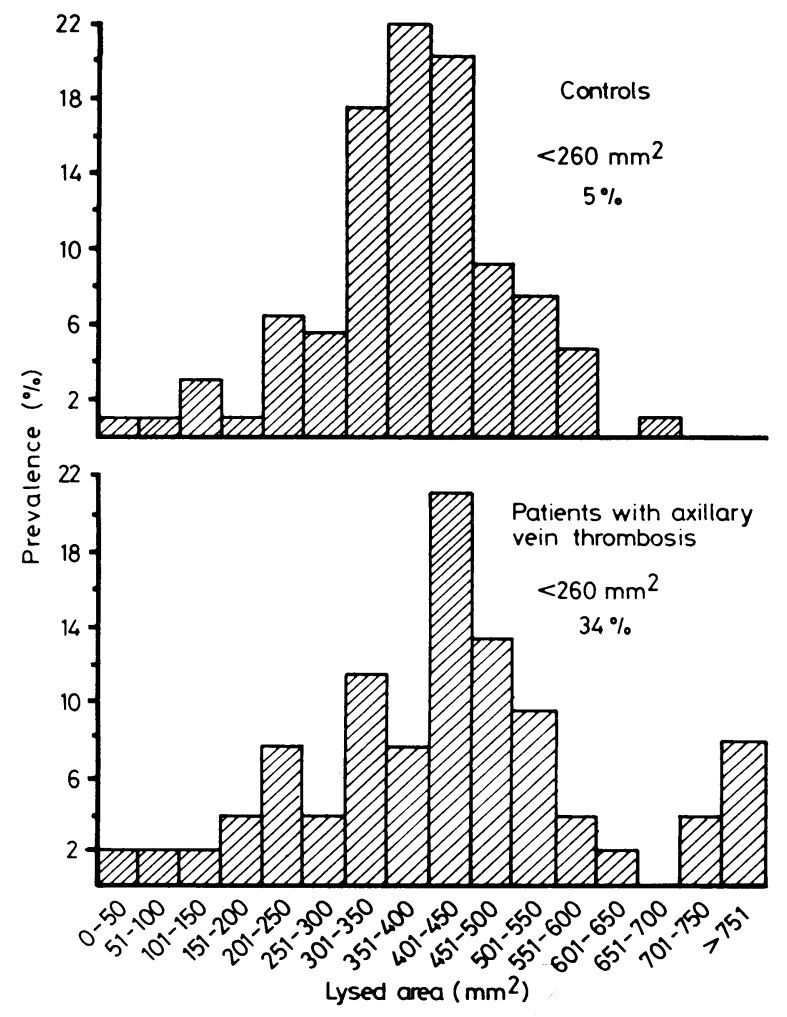

FIG 1-Fibrinolytic activity after venous occlusion in controls and patients with axillary vein thrombosis.

The fibrinolytic defence mechanism was studied in two ways: the capacity to release fibrinolytic activators from the vessel walls, and the fibrinolytic activity in vessel-wall biopsy specimens.

The release capacity of fibrinolytic activators from the vessel wall was measured as the local fibrinolytic activity obtained after venous occlusion of the arms for 20 minutes. Venous occlusion was obtained by applying a sphygmomanometer cuff around each arm and inflating the cuffs to a pressure midway between the systolic and diastolic pressures for 20 minutes. ${ }^{16}$ Blood samples for determination of the fibrinolytic activity were drawn immediately before the cuff was applied and immediately before it was deflated. The samples were drawn from an antecubital vein. Euglobulin precipitate was prepared from the plasma and the fibrinolytic activity measured on fibrin plates as described. ${ }^{16}$ The mean increase in the fibrinolytic activity in the samples from each arm was taken as a measure of the release capacity. The fibrinolytic activity after venous occlusion in the control subjects varied within the range $269-496 \mathrm{~mm}^{2}$ ( $95 \%$ confidence interval).

The fibrinolytic activity in vessel walls was determined histochemically using the technique of Todd as modified by Pandolfi. ${ }^{17} \mathrm{~A}$ segment of the distal part of the cephalic vein was excised under local anaesthesia (mepivacaine hydrochloride $0.5 \%$ ). After freezing, the vessel specimens were covered with a fibrin film containing plasminogen and incubated at $37^{\circ} \mathrm{C}$ for various periods. The size of the lytic zones formed in the fibrin film during incubation is taken as a measure of the fibrinolytic activity of the vessel wall. The activity is expressed in arbitrary units according to Pandolfi. ${ }^{17}$ The median value found in 60 healthy volunteers ( 31 women and 29 men, average age 37.0 years, range 19-79 years) was $7 \cdot 5$ arbitrary units (range 6-10).

\section{Results}

The fibrinolytic defence mechanism was examined in 53 of the 60 patients (figs 1 and 2). Fibrinolytic activity was less than $260 \mathrm{~mm}^{2}$ in 18 of the patients $(34 \%)$ and six of the 108 controls (5\%). Fibrinolytic activity within the vessel walls was less than six arbitrary units in 10 of the patients $(19 \%)$.

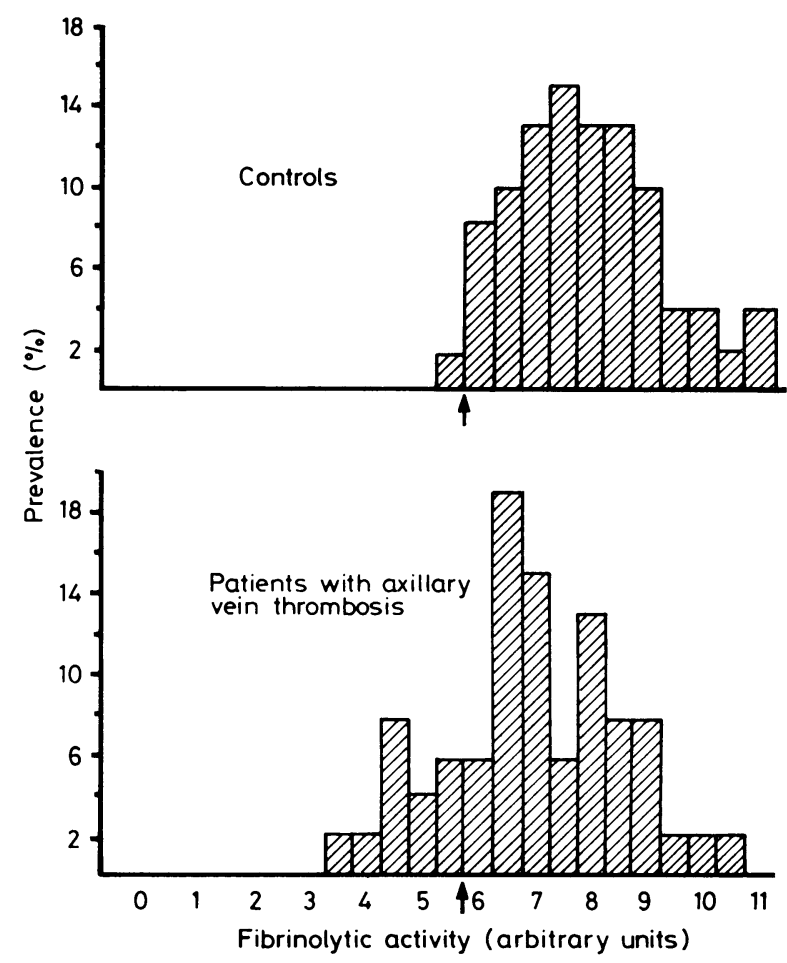

FIG 2-Fibrinolytic activity within the vessel wall in controls and patients with axillary vein thrombosis.

The release of fibrinolytic activity after venous occlusion on two consecutive days was low $\left(<260 \mathrm{~mm}^{2}\right)$ in 18 of the 53 patients $(34 \%)$. The fibrinolytic activity in the vessel wall was low (below six arbitrary units) in 10 of the patients $(19 \%)$. A decrease in the fibrinolytic defence mechanism, expressed as either a defective capacity to release fibrinolytic activity into the circulation of the venous occlusion or decreased fibrinolytic activity in the vessel wall, or both, was found in 26 patients ( 17 women and nine men; $49 \%$ ).

The development of fibrinolytic activity after venous occlusion was studied in both the post-thrombotic arm and the healthy arm in 17 of the 18 patients with a decreased release capacity. All 17 showed a low release capacity $\left(<260 \mathrm{~mm}^{2}\right)$ in both arms. Thus local factors in the thrombotic arm do not seem to influence the release capacity.

Of the 34 patients in whom factors supposed to be important for the development of thrombosis were found, 29 were examined for vesselwall fibrinolysis. Of these, $15(52 \%)$ also had an impaired fibrinolytic 
defence mechanism. A defective fibrinolytic defence mechanism was found in five out of nine women $(56 \%)$ taking contraceptive pills.

Factor VIII clotting activity was assayed in 57 patients. Of these, 22 had values between 160 and $250 \%$ (normal range $60-160 \%$ ) and four had excessively high values $(>250 \%$ ). Three of these four patients had a normal fibrinolytic defence mechanism. The fourth patient, however, had both a decreased release capacity and defective vesselwall fibrinolysis in addition to her greatly increased factor VIII clotting activity.

Factor VIII-related antigen was determined in 39 patients. It was increased to between 160 and $250 \%$ (normal range $60-160 \%$ ) in seven patients and to over $250 \%$ in only one patient. This last patient, who had developed his thrombosis during intensive care for hepatic coma, also had increased factor VIII clotting activity in addition to an impaired fibrinolytic defence mechanism.

Antithrombin III was determined immunochemically in 48 patients. All 48 showed normal values (normal range 69-120\%).

Inhibitors of the plasminogen activation were assayed in 56 patients using a clot lysis assay. ${ }^{11}$ Seven patients had increased values $(>140 \%)$. A normal fibrinolytic defence mechanism was found in three of these seven and a decreased mechanism in two. The two others were not examined for vessel-wall fibrinolysis or release capacity.

\section{Discussion}

The present series of patients with a deep venous thrombosis in the upper arm is one of the largest reported and the first in which detailed studies of coagulation and fibrinolysis have been carried out. The peak incidence occurred at the same age as has previously been reported, but the series differed from others by containing a preponderance of women. Furthermore, in only seven of the 60 patients $(12 \%)$ was preceding trauma or some special effort found. In three of the patients the thoracic outlet syndrome was a possible cause or contributing factor for the development of thrombosis. Other predisposing factors, found in some patients, were cancer and congestive heart failure. We should emphasise, however, that the patients were selected from the point of view that most had been referred from other parts of the country to our hospital. Possibly a higher proportion of patients whose disease pattern was unusual had been referred for special examination.

An impaired fibrinolytic defence mechanism was found in $49 \%$ of the patients, compared with $70 \%$ of patients with spontaneous deep venous thrombosis in the lower leg. ${ }^{10}$ The prevalence of this defect in our patients with deep venous thrombosis of the upper arm, however, is remarkably high and seems to indicate the existence of a previously unknown pathogenetic factor in this disease. In a control series described by Isacson and Nilsson ${ }^{10}$ no significant difference was found in the fibrinolytic release capacity between 18 people aged over 40 years compared with 42 aged below 40 years and there was no difference between the sexes. Therefore, the increased prevalence of a defective fibrinolytic defence mechanism in patients with axillary vein thrombosis does not seem to depend on any special distribution of sex or age.

Vessel-wall fibrinolysis was examined in 29 of the 34 patients in whom one or more possibly thrombogenic factors were found. In 15 of these 29 patients a defective fibrinolytic capacity was found. Among the other patients the corresponding figure was 14 out of 29. Thus there does not seem to be any increased risk to develop deep venous thrombosis in the arm when an additional thrombogenic factor such as trauma, infection, myocardial infarction, etc, is combined with defective vesselwall fibrinolysis.

The proportions of women taking the contraceptive pill in this series in all three age groups were about the same as those in the Swedish population as a whole. ${ }^{18}$ The higher proportion of women in our series of patients with deep venous thrombosis in the upper arm compared with previous series cannot, therefore, be explained by a more general use of contraceptive pills.

A decreased concentration of antithrombin III predisposes for deep venous thrombosis. ${ }^{1219}$ All patients in this series, however, had a normal antithrombin III concentration. In- creased inhibition of the plasminogen activation has also been reported as being associated with thrombotic disease. ${ }^{14}$ No substantial increase in this inhibitory activity, however, was seen in our patients. High concentrations of factor VIII, especially factor VIII-related antigen, have been suggested to be important in the aetiology of thrombotic disease. ${ }^{13}$ In our series only one patient had a substantial and selective increase in factor VIII-related antigen; such an increase does not, therefore, seem to be of any importance as a contributory factor.

Our findings confirm the multifactorial character of deep venous thrombosis in the upper arm. They also show that a deficient fibrinolytic capacity, known to be important for development of spontaneous deep venous thrombosis in the lower leg, is also important for deep venous thrombosis in the upper arm. Studies of fibrinolysis may therefore be important in evaluating these patients, particularly since deficient fibrinolytic capacity may now be restored to normal by medical treatment. ${ }^{20-22}$

This investigation was supported by grants from the Swedish Medical Research Council (B81-19X-05447-03; B81-19X-00087-17) and the Medical Faculty, University of Lund.

Requests for reprints should be addressed to: Professor Sven-Erik Bergentz, Department of Surgery, Malmö General Hospital, S-214 01 Malmö, Sweden.

\section{References}

${ }^{1}$ Adams JT, McEvoy RK, DeWeese JA. Primary deep venous thrombosis of upper extremity. Arch Surg 1965;91:29-42.

${ }^{2}$ French GE. Spontaneous thrombosis of axillary vein. $\mathrm{Br} \mathrm{Med} \mathcal{F} 1944$;ii : 277.

${ }^{3}$ Hughes ES. Venous obstruction in the upper extremity (Paget-Schroetter's syndrome). International Abstracts of Surgery 1949;88:89-127.

4 Paget J. Clinical lectures and essays. London: Longmans, Green and Co, 1875.

${ }^{5}$ von Schroetter L. Erkrankungen der Gefasse. In: Nothnagel CNH, ed. Handbuch der pathologie und therapie. Vienna: Holder, 1884.

${ }^{6}$ von Lenormont C. Le thrombose veineuse par effort. Presse Med 1912; $20: 761$.

7 Kleinsasser L. "Effort" thrombosis of axillary and subclavian veins: analysis of 16 personal cases and 56 cases collected from literature. Arch Surg 1949;59:258-74.

${ }^{8}$ Matas R. So-called primary thrombosis of axillary vein caused by strain: report of case, diagnosis, pathology and treatment. Am $\mathcal{F}$ Surg 1934 ;24: 642-66.

${ }^{9}$ Hughes ES. Venous obstruction in upper extremity. Br $\mathcal{F}$ Surg 1948 ;36 : 155-63.

${ }^{10}$ Isacson S, Nilsson IM. Defective fibrinolysis in blood and vein walls in recurrent "idiopathic" venous thrombosis. Acta Chir Scand 1972;138: 313-9.

${ }^{11}$ Hedner U, Nilsson IM, Jacobsen CD. Demonstration of low content of fibrinolytic inhibitors in individuals with high fibrinolytic capacity. Scand $\mathcal{F}$ Clin Lab Invest 1970;25:329-36.

12 Hedner U, Nilsson IM. Antithrombin III in a clinical material. Thromb Res $1973 ; 3: 631-41$.

${ }^{13}$ Holmberg L, Nilsson IM. Immunologic studies in haemophilia A. Scand F Haematol 1973;10:12-6.

14 Pandolfi M, Hedner U, Nilsson IM. Bilateral occlusion of the retinal veins in a patient with inhibition of fibrinolysis. Ann Ophthalmol 1970;2:481-4.

15 Nilsson IM, Kirkwood TBL, Barrowcliffe TW. In vivo recovery of factor VIII : a comparison of one-stage and two-stage assay methods. Thromb Haemost $1979 ; 42: 1230-9$.

16 Robertson BR, Pandolfi M, Nilsson IM. "Fibrinolytic capacity" in healthy volunteers as estimated from effect of venous occlusion of arms. Acta Chir Scand 1971;138:429-36.

17 Pandolfi M. Histochemistry and assay of plasminogen activator(s). Revue européenne d'études cliniques et biologiques 1972;17:254-60.

18 Astedt B, Isacson S, Nilsson IM, Pandolfi M. Thrombosis and oral contraceptives: possible predisposition. Br Med f 1973;iv:631-4.

19 Johansson L, Hedner U, Nilsson IM. Familiar antithrombin III deficiency as pathogenesis of deep venous thrombosis. Acta Med Scand 1978;204: 491-5.

${ }^{20}$ Hedner U, Nilsson IM, Isacson S. Effect of ethyloestrenol on fibrinolysis in the vessel wall. $\mathrm{Br} \mathrm{Med} \mathcal{F}$ 1976;ii:729-31.

${ }^{21}$ Nilsson IM, Hedner U, Isacson S. Phenformin and ethyloestrenol in recurrent venous thrombosis. Acta Med Scand 1975;198:107-13.

${ }^{22}$ Nilsson B, Nilsson IM, Hedner U. $\Delta$ 4-ethylestrenol in recurrent deep venous thrombosis. Acta Med Scand $1981 ; 209: 45-9$. 